\title{
Abnormal Centrosome Amplification and Aurora-A Activation in p53-deficient Cells
}

\author{
Eri Oda-Sato and Nobuyuki Tanaka
}

Department of Molecular Oncology, Institute of Gerontology, Nippon Medical School

a

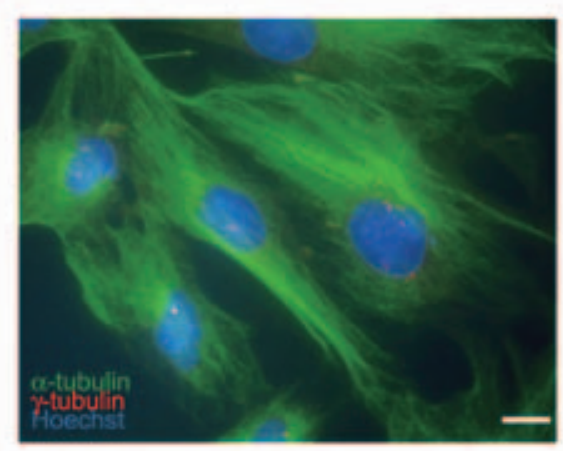

b
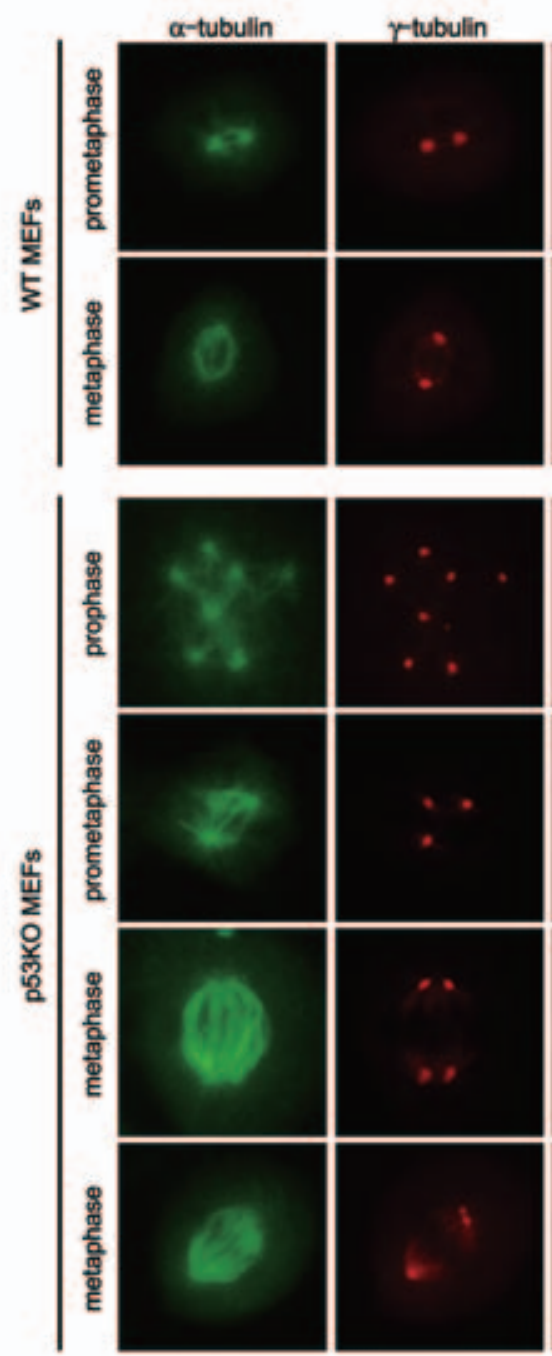

Hoechst
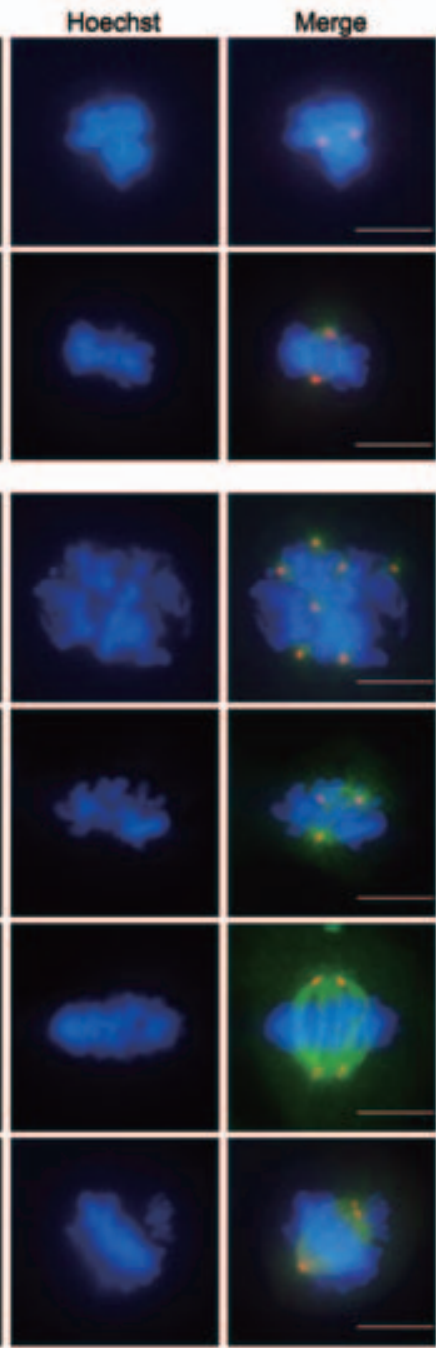

Fig. 1

The p53 tumor-suppressor gene is the most frequent target of genetic alterations in human cancer. Numerous studies have demonstrated that the loss of p53 function results in genetic instability, such as mutation, chromosomal translocation, and aneuploidy. It has been shown that the loss of p53 function leads to an abnormal centrosome amplification because of the deregulation of the centrosome duplication cycle. As shown in Figure 1a, centrosome hyperamplification (stained with anti- $\gamma$-tubulin antibody; red) was frequently observed in p53-deficient $\left(p 53^{-/-}\right)$mouse embryonic fibroblasts (MEFs) at interphase. During mitosis, centrosomes form spindle poles and play a vital role in bipolar spindle formation and accurate chromosomal segregation (Fig. 1b). In contrast, the mitotic $p 53^{-/-}$MEFs frequently displayed aberrant spindles, organized by multiple copies of

Correspondence to Eri Oda-Sato, Department of Molecular Oncology, Institute of Gerontology, Nippon Medical School, 1-396 Kosugi-cho, Nakahara-ku, Kawasaki, Kanagawa 211-8533, Japan 
a

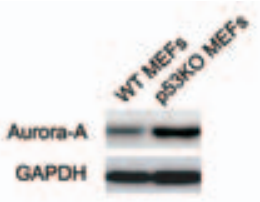

b

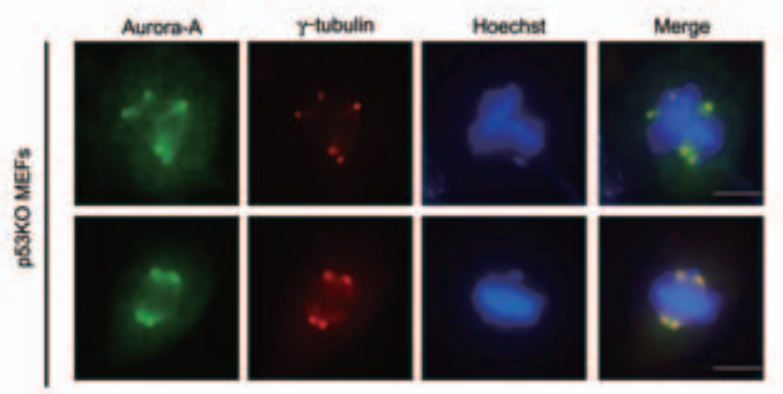

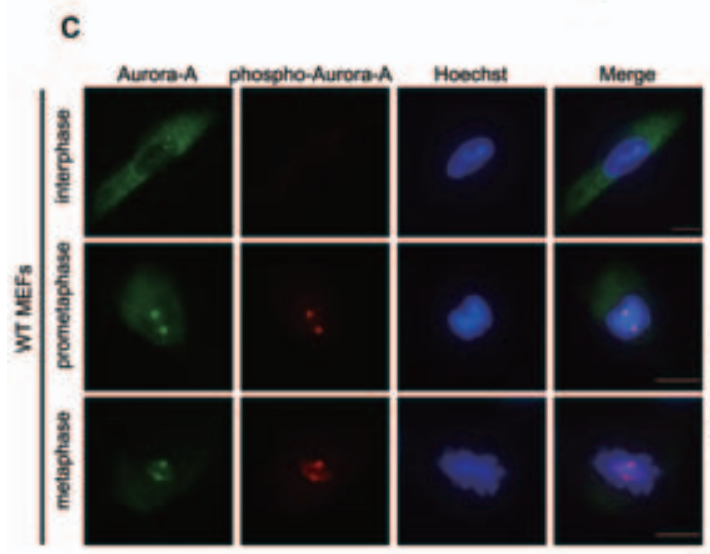
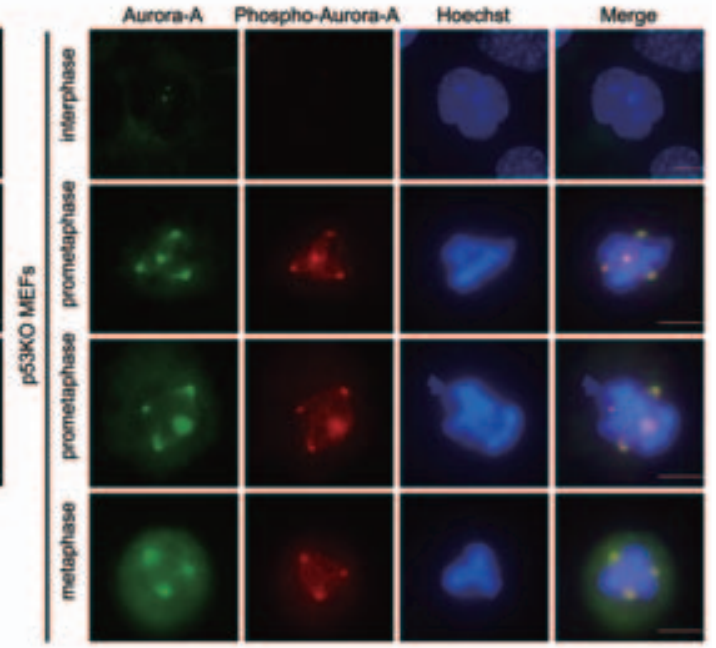

Fig. 2

centrosomes (Fig. 1b).

Aurora-A is a mitotic kinase that localizes to the centrosome and the bipolar mitotic spindle poles. It has been also shown that overexpression of Aurora-A induces centrosome amplification in cultured cells. We analyzed Aurora-A protein levels and found that those in $p 53^{-/-}$MEFs were higher than those in wild type (WT) MEFs (Fig. 2a). Moreover, Aurora-A was found to localize to abnormally amplified centrosomes in $p 53^{-/-}$ MEFs (Fig. 2b). We also found that Aurora-A is activated in abnormally amplified centrosomes in $p 53^{-/-} \mathrm{MEFs}$ (Fig. 2c). Therefore, these results suggest that activated Aurora-A caused by the loss of p53, "the guardian of the genome." is involved in abnormal centrosome amplification.

Fig. 1 (a) $p 53^{-/-}$MEFs at interphase were coimmunostained with anti- $\alpha$-tubulin antibody (green: Cell Signaling Technology) and anti- $\gamma$-tubulin antibody (red: Sigma). DNA was stained with Hoechst 33342 (blue: Invitrogen). Scale bars: $10 \mu \mathrm{m}$.

(b) Mitotic WT or $p 53^{-/-}$MEFs coimmunostained with anti- $\alpha$-tubulin antibody (green) and anti- $\gamma$ tubulin antibody (red). DNA was stained with Hoechst 33342 (blue). Scale bars: $10 \mu \mathrm{m}$.

Fig. 2 (a) Aurora-A protein levels in WT and $p 53^{-/-}$MEFs were determined by immunoblot analysis. (b) The mitotic $p 53^{-/-}$MEFs were coimmunostained with Aurora-A (green: Novus Biologicals, Inc) and anti- $\gamma$-tubulin antibody (red). DNA was stained with Hoechst 33342 (blue). Scale bars: $10 \mu \mathrm{m}$. (c) Mitotic WT or $p 53^{-/-}$MEFs were coimmunostained with anti-Aurora-A antibody (green) and antiphosphorylated Aurora-A antibody (red: This antibody was a gift from Dr. Urano, Nagoya University Graduate School of Medicine). DNA was stained with Hoechst 33342 (blue). Scale bars: $10 \mu \mathrm{m}$.

E-mail: eri@nms.ac.jp Journal Website (http://www.nms.ac.jp/jnms/) 